\title{
On the Informativeness of Dominant and Co-Dominant Genetic Markers for Bayesian Supervised Clustering
}

\author{
Gilles Guillot*,1 $^{*}$ and Alexandra Carpentier-Skandalis ${ }^{2}$
}

\author{
${ }^{I}$ Department of Informatics and Mathematical Modelling, Technical University of Denmark, 2800, Lyngby, \\ Copenhagen, Denmark; ${ }^{2}$ Centre for Ecological and Evolutionary Synthesis, Department of Biology, University of Oslo, \\ P.O. Box 1066 Blindern, 0316 Oslo, Norway
}

\begin{abstract}
We study the accuracy of a Bayesian supervised method used to cluster individuals into genetically homogeneous groups on the basis of dominant or codominant molecular markers. We provide a formula relating an error criterion to the number of loci used and the number of clusters. This formula is exact and holds for arbitrary number of clusters and markers. Our work suggests that dominant markers studies can achieve an accuracy similar to that of codominant markers studies if the number of markers used in the former is about 1.7 times larger than in the latter.
\end{abstract}

Keywords: Assigment method, multilocus genotype, SNP, AFLP, likelihood, Bayes estimator.

\section{BACKGROUND}

A common problem in population genetics consists in assigning an individual to one of $K$ populations on the basis of its genotype and information about the distribution of the various alleles in the $K$ populations. This question has received a considerable attention in the population genetics and molecular ecology literature [1-4] as it can provide important insight about gene flow patterns and migration rates. It is for example widely used in epidemiology to detect the origin of a pathogens or of their hosts (see e.g. [5-7] for examples) or in conservation biology and population management to detect illegal trans-location or poaching [8]. See [9] for a review of related methods.

In a statistical phrasing, assigning an individual to some known clusters is a supervised clustering problem. This requires to observe the genotype of the individual to be assigned and those of some individuals in the various clusters. For diploid organisms (i.e. organisms harbouring two copies of each chromosome), certain lab techniques allow one to retrieve the exact genotype of each individual. In contrast, for some markers it is only possible to say whether a certain allele $A$ (referred hereafter as to dominant allele) is present or not at a locus. In this case, one can not distinguish the heterozygous genotype $A a$ from the homozygous genotype $A A$ for the dominant allele. The former type of markers are said to be codominant while the latter are said to be dominant. It is clear that the the second genotyping method incurs a loss of information. The consequence of this loss of information has been studied from an empirical point of view [10] but it has never been studied on a theoretical basis. The choice to use one type of

*Address correspondence to this author at the Department of Informatics and Mathematical Modelling, Technical University of Denmark, 2800, Lyngby, Copenhagen, Denmark; Tel: +4545253321;

E-mail: gigu@imm.dtu.dk markers for empirical studies is therefore often motivated mostly by practical considerations rather than by an objective rationale $[11,12]$. The objective of the present article is to compare the accuracy achieved with dominant and codominant markers when they are used to perform supervised clustering and to derive some recommendations about the number of markers required to achieve a certain accuracy. Dominant markers are essentially bi-allelic in the sense that they record the presence of the absence of a certain allele. We are not concerned here by the relation between informativeness and the level of polymorphisms (cf $[13,14]$ for references on this aspect). We therefore focus on bi-allelic dominant and co-dominant markers. Hence our study is representative of Amplified Fragment Length Polymorphism (AFLP) and Single Nucleotide Polymorphism (SNP) markers, which are some of the most employed markers in genetics.

\section{INFORMATIVENESS OF DOMINANT AND CO- DOMINANT MARKERS}

\subsection{Cluster Model}

We will consider here the case of diploid organisms at $L$ bi-allelic loci. We denote by $z=\left(z_{l}\right)_{l=1, \ldots, L}$ the genotype of an individual. We denote by $f_{k l}$ the frequency of allele $A$ in cluster $k$ at locus $l$ We assume that each cluster is at HardyWeinberg equilibrium (HWE) at each locus. HWE is defined as the conditions under which the allele carried at a locus on one chromosome is independent of the allele carried at the same locus on the homologous chromosome. This situation is observed at neutral loci when individuals mate at random in a cluster. Denoting by $z_{l}$ the number of copies of allele $A$ carried by an individual, we have: For co-dominant markers, this can be expressed as

$$
p\left(z_{l}=2 \mid f\right)=f_{l}^{2}
$$




$$
\begin{aligned}
& p\left(z_{l}=1 \mid f\right)=2 f_{l}\left(1-f_{l}\right) \\
& p\left(z_{l}=0 \mid f\right)=\left(1-f_{l}\right)^{2}
\end{aligned}
$$

For dominant markers, $z_{l}$ is equal to 0 or 1 depending on whether a copy of allele $A$ is present in the genotype of the individual. Under HWE we have:

$$
\begin{aligned}
& p\left(z_{l}=1 \mid f\right)=f_{l}^{2}+2 f_{l}\left(1-f_{l}\right) \\
& p\left(z_{l}=0 \mid f\right)=\left(1-f_{l}\right)^{2}
\end{aligned}
$$

In addition to HWE, we also assume that the various loci are at linkage equilibrium (henceforth HWLE), i.e. that the probability of a multilocus genotype is equal to the product of probabilities of single-locus genotypes: $p\left(z_{1}, \ldots ., z_{L}\right)=\prod_{l} p\left(z_{l}\right)$. We assume that the individual to be classified has origin in one of the $K$ clusters (no admixture).

\subsection{Sampling Model}

We will measure the accuracy of a classifying rule for a given type of markers by the probability to assign correctly an individual with unknown origin. We are interested in deriving results that are independent (i) on the particular origin $c$ of the individual to be classified (ii) on the genotype $z$ of this individual and (iii) on the allele frequencies $f$ in the various clusters. We will therefore derive results that are conditional on $c, z$ and $f$ and then compute Bayesian averages under suitable prior distributions. The mechanism assumed in the sequel is as follows

1. The individual has origin in one of the $K$ clusters. This origin is unknown and all origins are equally likely. We therefore assume a uniform prior for $c$ on $\{1, \ldots, K\}$.

2. In each cluster, for each locus the allele frequencies follow a Dirichlet $(1,1)$ distribution with independent across clusters and loci.

3. Conditionally on $c$ and $f$, the probability of the genotype of the individual is given by equations (1)-(3) or (4)-(5), i.e we assume that the individual has been sampled at random among all individuals in his cluster of origin.

\subsection{Accuracy of Assignments Under a Maximum Likelihood Principle}

We consider an individual of unknown origin $c$ with known genotype $z$ with potential origin in $K$ clusters with known allele frequencies. Following a maximum likelihood principle, it is natural to estimate $c$ as the cluster label for which the probability of observing this particular genotype is maximal. Formally: $c^{*}=\operatorname{Argmax}_{k} p\left(z \mid c=k, f_{k}\right)$. This assignment rule is deterministic, but whether the individual is correctly assigned will depend on its genotype and on cluster allele frequencies. Randomising these quantities and averaging over all possible values, we can derive a generic formula for the probability of correct assignment $p^{M L A}$ as

$$
p^{M L A}=\int_{\varphi} \sum_{\zeta} \max _{\gamma} p(c=\gamma, z=\zeta \mid f=\varphi) d p(\varphi)
$$

See section A in appendix for details. This formula is of little practical use and deriving some more explicit expression for arbitrary value of $K$ and $L$ seems to be out of reach. However, for $K=2$ and $L=1$, under the assumptions that the individual has a priori equally likely ancestry in each cluster and that each $f_{k}$ has a Dirichlet distribution with parameter $(1, \ldots, 1)$ (flat). We get

$p_{c}^{M L A}(K=2, L=1)=17 / 24$ for codominant markers

and

$p_{d}^{M L A}(K=2, L=1)=16 / 24$ for dominant markers

Because of the lack of practical usefulness of eq. (6), we now define an alternative rule for assignment that is similar in spirit to maximum likelihood but also leads to more tractable equations.

\subsection{Accuracy of Assignments Under a Stochastic Rule}

Considering the collection of likelihood values $p\left(z \mid c=k, f_{k}\right)$ for $k=1, \ldots, K$, following [15], we define a stochastic assignment (SA) rule by assigning the individual to a group at random with probabilities proportional to $p\left(z \mid c=k, f_{k}\right)$. In words, an individual with genotype $z$ is randomly assigned to cluster $k$ with a probability proportional to the probability to observe this genotype in cluster $k$. The rationale behind this rule is that high values of $p\left(z \mid c=k, f_{k}\right)$ indicate strong evidence of ancestry in group $k$ but do not guarantee against miss-assignments. To derive the probability of correct assignment, we first consider that the allele frequencies are known, and then account for the uncertainty about these frequencies by Bayesian integration. The use of a Bayesian framework is motivated by the fact that (i) there is a genuine uncertainty on allele frequencies which can not be overlooked, and (ii) under some fairly mild assumptions, allele frequencies are known to be Dirichlet distributed (possibly with a degree of approximation see e.g. $[16,17])$. Refer to $[18]$ for further discussion of the Bayesian paradigm in population genetics.

We now give our main results regarding this clustering rule.

For bi-allelic loci and denoting by $p_{c}^{S A}$ the probability of correct assignment using codominant markers we have:

$$
p_{c}^{S A}(K, L)=\frac{1}{1+(K-1)(5 / 8)^{L}}
$$

For bi-allelic loci and denoting by $p_{d}^{S A}$ the probability of correct assignment using dominant markers is

$$
p_{d}^{S A}(K, L)=\frac{1}{1+(K-1)(25 / 33)^{L}}
$$

\section{IMPLICATIONS}

Our investigations considered bi-allelic loci and are therefore representative of AFLP and SNP markers which 
are some of the most employed markers in genetics. In this context, for supervised clustering, our main conclusions are that (i) codominant markers are more accurate than dominant markers, (ii) the difference of accuracy decreases toward 0 as the number of markers $L$ increases, (iii) $L_{d}$ dominant markers can achieve an accuracy even higher than that of $L_{c}$ codominant markers as long as the numbers of loci used satisfy $L_{d} \geq \lambda L_{c}$ where $\lambda=\ln (5 / 8) / \ln (25 / 33) \approx 1.69$.

The figures reported have to be taken with a grain of salt as they may depend on some specific aspects of the models considered. For example, the model considered here assumes independence of allele frequencies across clusters. This assumption is relevant in case of populations displaying low migration rates and low amount of shared ancestry. When one of these assumptions is violated, an alternative parametric model based on the Dirichlet distribution that accounts for correlation of allele frequencies across population is often used (see [16] and references therein). It is expected that the accuracy obtained with both markers would be lower under this model. Besides, the present study does not account for ascertainment bias [19-22], an aspect that might affect the results but is notoriously difficult to deal with. However, it is important to note that the conditions considered in the present study were the same for dominant and codominant markers so that results should not be biased toward one type of marker. Our global result about the relative informativeness of dominant and co-dominant markers contrasts with the common belief that dominant markers are expedient one would resort to when co-dominant markers are not available (see [12] for discussions).

A comparison of dominant and codominant markers for unsupervised clustering has been carried out [23]. This study based on simulations suggests that the loss of accuracy incurred by dominant markers in unsupervised clustering is much larger than for supervised clustering. This is presumably explained by the fact that in case of HWLE clusters, supervised clustering seeks to optimise a criterion based on allele frequencies only. This contrasts with unsupervised clustering which seeks to optimise a criterion based on allele frequencies and HWLE. A similar theoretical analysis of unsupervised clustering algorithm similar to the present study would be valuable but we anticipate that it would present more difficulties.

\section{ACKNOWLEGEMENT}

This work has been supported by Agence Nationale de la Recherche grant ANR-09-BLAN-0145-01.

\section{APPENDIX}

\section{A. Supervised Clustering with a Maximum Likelihood Principle}

We consider the setting where the unknown ancestry $c$ of an individual with genotype $z$ is estimated by $c^{*}=\operatorname{Argmax}_{c} p(z \mid c, f)$. As this estimator is a deterministic function of $z$ we denote it by $c_{z}^{*}$ for clarity in the sequel. Consider for now that the allele frequencies $f$ are known to be equal to some $\varphi$. Under this setting, randomness comes from the sampling of $c$ and then from the sampling of $z \mid(c, f)$. We are concerned with the event $\varepsilon$ defined as

$$
\varepsilon=\{\text { the individual is correctly assigned }\}
$$

Applying the total probability formula, we can write

$$
p(\varepsilon \mid f=\varphi)=\sum_{\gamma} \sum_{\zeta} p(\varepsilon, c=\gamma, z=\zeta \mid f=\varphi)
$$

In the sum over $\gamma$, only one term is not equal to 0 , this is the term for $\gamma=c^{*}$, hence

$$
\begin{aligned}
p(\varepsilon \mid f= & \varphi)=\sum_{\zeta} p\left(\varepsilon, c=c_{\zeta}^{*}, z=\zeta \mid f=\varphi\right) \\
& =\sum_{\zeta} p\left(c=c_{\zeta}^{*}, z=\zeta \mid f=\varphi\right) \\
& =\sum_{\zeta} p\left(c=c_{\zeta}^{*} \mid f=\varphi\right) p\left(z=\zeta \mid c=c_{\zeta}^{*}, f=\varphi\right) \\
& =\sum_{\zeta} p\left(c=c_{\zeta}^{*}\right) p\left(z=\zeta \mid c=c_{\zeta}^{*}, f=\varphi\right)
\end{aligned}
$$

Assuming that the individual has a priori equally likely ancestry in each cluster, i.e. assuming a uniform distribution for the class variable $c$, we get

$$
p(\varepsilon \mid f=\varphi)=K^{-1} \sum_{\zeta} p\left(z=\zeta \mid c=c_{\zeta}^{*}, f=\varphi\right)
$$

By definition, $c_{z}^{*}$ satisfies $p\left(z \mid c_{z}^{*}, f\right)=\max _{\gamma} p(z \mid c=\gamma, f)$, hence

$$
\begin{aligned}
p(\varepsilon \mid f=\varphi) & =K^{-1} \sum_{\zeta} \max _{\gamma} p(z=\zeta \mid c=\gamma, f=\varphi) \\
& =\sum_{\zeta} \max _{\gamma} p(c=\gamma) p(z=\zeta \mid c=\gamma, f=\varphi) \\
& =\sum_{\zeta} \max _{\gamma} p(c=\gamma, z=\zeta \mid f=\varphi)
\end{aligned}
$$

We seek an expression of the probability of correct assignment that does not depend on particular values of allele frequencies. This can be obtained by integrating over allele frequencies, namely

$$
\begin{aligned}
& p(\varepsilon)=\int_{\varphi} p(\varepsilon \mid f=\varphi) d p(\varphi) \\
& =\int_{\varphi} \sum_{\zeta} \max _{\gamma} p(c=\gamma, z=\zeta \mid f=\varphi) d p(\varphi)
\end{aligned}
$$

Note that identity (21) holds for any number of cluster $K$, any number of loci $L$ and any type of markers (dominant vs. codominant).

We now consider a two-cluster problem in the case where the genotype of an individual has been recorded at a single bi-allelic locus. We denote by $f_{1}$ (resp. $f_{2}$ ) the frequency of allele $A$ in cluster 1 (resp. cluster 2).

\section{A.1. Codominant Markers}

There are only three genotypes: $A A, A a$ and $a a$. Denoting by $f_{k}$ the frequency of allele $A$ in cluster $k$ and 
conditionally on $f_{k}$, these three genotypes occur in cluster $k$ with probabilities $f_{k}^{2}, 2 f_{k}\left(1-f_{k}\right)$ and $\left(1-f_{k}\right)^{2}$, and equation (21) can be simplified as

$p(\varepsilon)=\int_{\varphi} p(c)\left[\max _{\gamma} f_{\gamma}^{2}+2 \max _{\gamma} f_{\gamma}\left(1-f_{\gamma}\right)+\max _{\gamma}\left(1-f_{\gamma}\right)^{2}\right] d p(\varphi)$

We need to derive the distribution of $\max _{\gamma} f_{\gamma}^{2}$ and of $\max _{\gamma} f_{\gamma}\left(1-f_{\gamma}\right)$. Assuming a flat Dirichlet distribution for $f_{k}$, elementary computations give:

$p\left(\max _{k} f_{k}^{2}<x\right)=x$

i.e $\max _{k} f_{k}^{2}$ follows a uniform distribution on $[0,1]$ so that

$\mathrm{E}\left(\max _{k} f_{k}^{2}\right)=1 / 2$

Besides, we also get

$p\left(\max _{k} f_{k}\left(1-f_{k}\right)<x\right)=(1-\sqrt{1-4 x})^{2}$

and deriving

$\frac{d p}{d x}\left(\max _{k} f_{k}\left(1-f_{k}\right)<x\right)=4 \frac{1-\sqrt{1-4 x}}{\sqrt{1-4 x}}$

Integrating by part, we get

$\mathbb{E}\left(\max _{k} f_{k}\left(1-f_{k}\right)\right)=\int_{0}^{1 / 4} 4 x \frac{1-\sqrt{1-4 x}}{\sqrt{1-4 x}} d x=5 / 24$

Eventually

$p(\varepsilon)=17 / 24$

which proves equation (7).

\section{A.2. Dominant Markers}

For a single locus, there are two genotypes $A$ and $a$. Conditionally on $f_{k}$, these two genotypes are observed in cluster $k$ with probabilities $1-f_{k}^{2}$ and $f_{k}^{2}$. Equation (21) can be simplified here as

$p(\varepsilon)=\int_{\varphi} p(c)\left[\max _{\gamma} f_{\gamma}^{2}+\max _{\gamma}\left(1-f_{\gamma}^{2}\right)\right] d p(\varphi)$

We now need the density of $1-f_{\gamma}^{2}$

$$
\begin{aligned}
& p\left(\max _{k}\left(1-f_{k}^{2}\right)<x\right)=(1-\sqrt{1-x})^{2} \\
& \frac{d p}{d x}\left(\max _{k}\left(1-f_{k}^{2}\right)<x\right)=\frac{1}{\sqrt{1-x}}-1
\end{aligned}
$$

and

$\mathbb{E}\left(\max _{k}\left(1-f_{k}^{2}\right)\right)=\int_{0}^{1} x\left(\frac{1}{\sqrt{1-x}}-1\right) d x=5 / 6$

Eventually we get

$p(\varepsilon)=16 / 24$

which proves equation (8).

\section{B. STOCHASTIC ASSIGNMENT RULE}

The maximum likelihood assignment rule considered above is not tractable for arbitrary values of $K$ and $L$ (cf. eq. (21)). In particular, a difficulty arises from the maximisation involved. We consider here an assignment rule that does not involve maximisation. The unknown ancestry $c$ of an individual with genotype $z$ is predicted by a random variable $c^{*}$ with values in $\{1, \ldots, K\}$ and such that $p\left(c^{*}=k \mid z, f\right) \propto p(z \mid c=k, f)$. As in the previous sections, we first consider that the allele frequencies are known, however we skip this dependence in the notation at the beginning for clarity. We will account for the uncertainty about these frequencies later by Bayesian in integration. In this setting, the structure of conditional probability dependence can be represented by a directed acyclic graph as in the on left-hand side of Fig. (1).

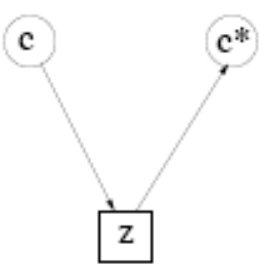

(a)

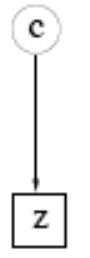

(b)
Fig. (1). Directed acyclic graph for our stochastic assignment rule (left) and for an alternative scheme (right). All downward arrows represent the same conditional dependence given by our likelihood model. Upward arrow represents the reverse probability dependence.

We are concerned with evaluating the probability of event $\varepsilon$ defined as

$$
\varepsilon=\{\text { the individual is correctly assigned }\}
$$

i.e. $\varepsilon=\left\{c=c^{*}\right\}$. We denote by $p_{a}$ (resp. $p_{b}$ ) probabilities under the two conditional dependence structure of Fig. (1). Some elementary computations show that $p(\varepsilon)$ can be expressed in terms of a probability in the model of the righthand-side of the DAG in Fig. (1), namely:

$$
p_{a}\left(c=c^{*}\right)=p_{b}\left(c=c^{\prime} \mid z=z^{\prime}\right)
$$

The left-hand-side of this expression can be written as

$$
p_{b}\left(c=c^{\prime} \mid z=z^{\prime}\right)=p_{b}\left(c=c^{\prime}, z=z^{\prime}\right) / p_{b}\left(z=z^{\prime}\right)
$$

It is more convenient to manipulate this expression than $p_{b}\left(c=c^{*}\right)$. We will to use it to evaluate $p_{a}(\varepsilon)$.

\section{B.1. Codominant Markers}

We assume that the individual has a priori equally likely ancestry in each cluster. We slightly change the notation denoting by $z_{l}$ the count of allele $A$ at locus $l$ for the individual to be assigned. Then making the dependence on $f$ explicit in the notation, we have 


$$
\begin{aligned}
p_{b}\left(c=c^{\prime}, z=z^{\prime} \mid f\right) & =\sum_{z} \sum_{k} p_{b}^{2}(c, z \mid f) \\
& =\sum_{z} \sum_{k}\left[\frac{1}{K} \prod_{l} f_{k}^{z_{l}}\left(1-f_{k}\right)^{2-z_{l}}\left(2-\delta_{z_{l}}^{1}\right)\right]^{2}
\end{aligned}
$$

where $\delta_{z_{l}}^{1}$ denotes the Kronecker symbol that equals 1 if $z_{l}=1$ and 0 otherwise.

Accounting for uncertainty about $f$ by integration, we get

$$
\begin{aligned}
& p_{b}\left(c=c^{\prime}, z=z^{\prime}\right)=\int_{f} p_{b}\left(c=c^{\prime}, z=z^{\prime} \mid f\right) d f \\
& =\int_{f} \sum_{z} \sum_{k}\left[\frac{1}{K} \prod_{l} f_{k}^{z_{l}}\left(1-f_{k}\right)^{2-z_{l}}\left(2-\delta_{z_{l}}^{1}\right)\right]^{2} d f
\end{aligned}
$$

Among the terms enumerated in the sum over $z$ above, let us consider a generic term $z$ for which the number of loci having exactly $h$ heterozygous genotypes. The term corresponding to such a genotype $z$ in the sum above can be written

$\sum_{k} \frac{1}{K^{2}} 2^{2 h}\left[\int_{f} f^{2}(1-f)^{2} d f\right]^{h}\left[\int_{f} f^{4} d f\right]^{L-h}$

Denoting by $C_{L}^{h}$ the binomial coefficient, there are $C_{L}^{h} 2^{L-h}$ such terms. Equation (39) becomes

$p_{b}\left(c=c^{\prime}, z=z^{\prime}\right)=\sum_{h} \sum_{k} C_{L}^{h} 2^{L-h} \frac{1}{K^{2}} 2^{2 h}\left[\int_{f} f^{2}(1-f)^{2} d f\right]^{h}\left[\int_{f} f^{4} d f\right]^{L-h}$

Assuming a flat Dirichlet distribution for the allele frequencies, we get

$p_{b}\left(c=c^{\prime}, z=z^{\prime}\right)=\frac{1}{K}\left(\frac{8}{15}\right)^{L}$

We now need to evaluate $p_{b}\left(z=z^{\prime}\right)$, but since

$p_{b}\left(z=z^{\prime} \mid f\right)=\sum_{z} p_{b}^{2}(z \mid f)=\sum_{z}\left(\sum_{k} p_{b}(k, z \mid f)\right)^{2}$,

$p_{b}\left(z=z^{\prime}\right)=\int_{z} \sum_{z} p_{b}^{2}(z \mid f)=\sum_{z}\left(\sum_{k} p_{b}(k, z \mid f)\right)^{2}$

$=\int_{z} \sum_{z}\left(\sum_{k} p_{b}(k, z \mid f)^{2}+\sum_{k \neq k^{\prime}} p_{b}(k, z \mid f) p_{b}\left(k^{\prime}, z \mid f\right)\right)$

$=p_{b}\left(c=c^{\prime}, z=z^{\prime}\right)+$

$\sum_{h} C_{L}^{h} 2^{L-h}\left(\sum_{k \neq k^{\prime}} 2\left(\frac{1}{K^{2}} 2^{2 h}\left[\int_{f} f(1-f) d f\right]^{2 h}\left[\int_{f} f d f\right]^{4(L-h)}\right)\right)$

$=\frac{1}{K}\left(\frac{8}{15}\right)^{L}+\frac{K-1}{K} \frac{1}{3^{L}}$

Eventually,

$$
p(\varepsilon)=\frac{1}{1+(K-1)\left(\frac{5}{8}\right)^{L}}
$$

which proves equation (9).

\section{B.2. Dominant Markers}

We still have

$p_{b}\left(c=c^{\prime}, z=z^{\prime}\right)=\int_{f} \sum_{z} \sum_{k}\left[\frac{1}{K} \prod_{l} f_{k}^{z_{l}}\left(1-f_{k}\right)^{2-z_{l} l}\left(2-\delta_{z_{l}}^{1}\right)\right]^{2} d f$

For a generic genotype $z$ in the sum above, let us denote by $\gamma$ the number of loci carrying exactly one copy of the recessive allele, then

$p_{b}\left(c=c^{\prime}, z=z^{\prime}\right)=\sum_{r} \sum_{k} C_{r}^{l} \frac{1}{K^{2}}\left[\int_{f} f_{k}^{4} d f\right]^{r}\left[\int_{f}\left(1-f_{k}^{2}\right)^{2} d f\right]^{L-r}$

$=\sum_{r} \sum_{k} C_{r}^{l} \frac{1}{K^{2}}\left(\frac{1}{5}\right)^{L}\left(\frac{8}{3}\right)^{L-r}$

$=\frac{1}{K}\left(\frac{11}{15}\right)^{L}$

Moreover, by arguments similar to those used for codominant markers, we get

$$
p_{b}\left(z=z^{\prime}\right)=\frac{1}{K}\left(\frac{11}{15}\right)^{L}+\frac{K-1}{K}\left(\frac{5}{9}\right)^{L}
$$

And we get

$p_{b}\left(z=z^{\prime}\right)=\frac{1}{K}\left(\frac{11}{15}\right)^{L}+\frac{K-1}{K}\left(\frac{5}{9}\right)^{L}$

Eventually,

$$
p(\varepsilon)=\frac{1}{1+(K-1)\left(\frac{25}{33}\right)^{L}}
$$

which proves equation (10).

\section{REFERENCES}

[1] B. Rannala, and J. Mountain "Detecting immigration by using multilocus genotypes," Proceedings of the National Academy of Sciences USA, vol. 94, pp. 9197-9201, 1997.

[2] J. Cornuet, S. Piry, G. Luikart, A. Estoup, and M. Solignac, "New methods employing multilocus genotypes to select or exclude populations as origins of individuals," Genetics, vol. 153, pp. 1989-2000, 1999.

[3] D. Paetkau, R. Slade, M. Burdens, and A. Estoup, "Genetic assignment methods for the direct, real-time estimation of migration rate: a simulation-based exploration of accuracy and power," Molecular Ecology, vol. 15, pp. 55-65, 2004.

[4] A. Piry, S. Alapetite, J. Cornuet, D. Paetkau, L. Baudoin, and A. Estoup, "Geneclass2: A software for genetic assignment and firstgeneration migrant detection," Journal of Heredity, vol. 95, no. 6 , pp. 536-539, 2004.

[5] P. Gladieux, X. Zhang, D. Afoufa-Bastien, R. V. Sanhueza, M Sbaghi, , and B. L. Cam, "On the origin and spread of the scab disease of apple: Out of central Asia," PLoS One, vol. 3, no. 1, p. e1455, 2008

[6] A. P. de Rosas, E. Segura, L. Fichera, and B. García, "Macrogeographic and microgeographic genetic structure of the chagas disease vector triatoma infestans (hemiptera: reduviidae) 
from Catamarca, Argentina," Genetica, vol. 133, no. 3, pp. 247260, 2008.

[7] A. Bataille, A. A. Cunningham, V. Cedeño, L. Patiño, A. Constantinou, L. Kramer, and S. J. Goodman, "Natural colonization and adaptation of a mosquito species in Galápagos and its implications for disease threats to endemic wildlife," Proceedings of the National Academy of Sciences, vol. 106, no. 25, pp. 10230-10235, 2009.

[8] S. Manel, P. Berthier, and G. Luikart, "Detecting wildlfe poaching: identifying the origin of individuals with Bayesian assignment test and multilocus genotypes," Conservation Biology, vol. 13, no. 3, pp. $650-659,2002$.

[9] S. Manel, O. Gaggiotti, and R.Waples, "Assignment methods: matching biological questions with appropriate techniques," Trends in Ecology and Evolution, vol. 20, pp. 136-142, 2005.

[10] D. Campbell, P. Duchesne, and L. Bernatchez, "AFLP utility for population assignment studies: analytical investigation and empirical comparison with microsatellites," Molecular Ecology, vol. 12, pp. 1979-1991, 2003.

[11] C. Schlotterer, "The evolution of molecular markers - just a matter of fashion?" Nature Review Genetics, vol. 5, pp. 63-69, 2004.

[12] A. Bonin, D. Ehrich, and S. Manel, "Statistical analysis of amplified fragment length polymorphism data: a toolbox for molecular ecologists and evolutionists," Molecular Ecology, vol. 16, no. 18, pp. 3737-3758, 2007.

[13] N. Rosenberg, T. Burke, K. Elo, M. Feldman, P. Friedlin, M. Groenen, J. Hillel, A. Maki- Tanila, M. Tixier-Boichard, A. Vignal, $\mathrm{K}$. Wimmers, and S. Weigend, "Empirical evaluation of genetic clustering methods using multilocus genotypes from 20 chicken breeds," Genetics, vol. 159, pp. 699-713, 2001.
[14] S. T. Kalinowski, "Do polymorphic loci require larger sample sizes to estimate genetic dis2 tances?" Heredity, vol. 94, pp. 33-36, 2005.

[15] N. Rosenberg, L. Li, R. Ward, and J. K. Pritchard, "Informativeness of genetic markers for inference of ancestry," American Journal of Human Genetics, vol. 73, pp. 1402-1422, 2003.

[16] G. Guillot, "Inference of structure in subdivided populations at low levels of genetic differentiation. The correlated allele frequencies model revisited," Bionformatics, vol. 24, pp. 2222-2228, 2008.

[17] O. Gaggiotti, and M. Foll, "Quantifying population structure using the F-model," Molecular Ecology Resources, vol. 10, no. 5, p. 821830, 2010.

[18] M. A. Beaumont, and B. Rannala, "The Bayesian revolution in genetics,"Nature Review Genetics, vol. 5, pp. 251-261, 2005.

[19] R. Nielsen, and J. Signorovitch, "Correcting for ascertainment biases when analyzing SNP data: applications to the estimation of linkage quilibrium," Theoretical Population Biology, vol. 63, pp. 245-255, 2003

[20] R. Nielsen, M. Hubisz, and A. Clark, "Reconstituting the frequency spectrum of ascertained single-nucleotide polymorphism data," Genetics, vol. 168, pp. 2373-2382, 2004.

[21] M. Foll, M. Beaumont, and O. Gaggiotti, "An approximate Bayesian computation approach to overcome biases that arise when using AFLP markers to study population structure," Genetics, vol. 179, pp. 927-939, 2008.

[22] G. Guillot, and M. Foll, "Accounting for the ascertainment bias in Markov chain Monte Carlo inferences of population structure," Bioinformatics, vol. 25, no. 4, pp. 552-554, 2009.

[23] G. Guillot, and F. Santos, "Using AFLP markers and the Geneland program for the inference of population genetic structure," Molecular Ecology Resources, vol. 10, pp. 1082-1084, 2010.

(c) Guillot and Carpentier-Skandalis; Licensee Bentham Open.

This is an open access article licensed under the terms of the Creative Commons Attribution Non-Commercial License (http://creativecommons.org/licenses/by-nc/3.0/) which permits unrestricted, non-commercial use, distribution and reproduction in any medium, provided the work is properly cited. 